\title{
Frequency-domain characterization of random demodulation analog-to-information converters
}

\author{
Doris Bao, Pasquale Daponte, Luca De Vito, Sergio Rapuano \\ Department of Engineering, University of Sannio, Piazza Roma, 21, 82100 Benevento, Italy
}

\section{ABSTRACT}

The paper aims at proposing test methods for Analog-to-Information Converters (AICs).

In particular, the objective of this work is to verify if figures of merit and test methods, currently defined in standards for traditional Analog-to-Digital Converters, can be applied to AICs based on the random demodulation architecture.

For this purpose, an AIC prototype has been designed, starting from commercially available integrated circuits. A simulation analysis and an experimental investigation have been carried out to study the additional influencing factors such as the parameters of the reconstruction algorithm. Results show that standard figures of merit are in general capable of describing the performance of AICs, provided that they are slightly modified according to the proposals reported in the paper. In addition, test methods have to be modified in order to take into account the statistical behavior of AICs.

\section{Section: RESEARCH PAPER}

Keywords: Analog-to-Information Converter, Compressive sampling, Testing, Frequency domain.

Citation: Doris Bao, Pasquale Daponte, Luca De Vito, Sergio Rapuano, Frequency-domain characterization of random demodulation analog-to-information converters, Acta IMEKO, vol. 4, no. 1, article 17, February 2015, identifier: IMEKO-ACTA-04 (2015)-01-17

Editor: Paolo Carbone, University of Perugia

Received January $14^{\text {th }}, 2014$; In final form April $4^{\text {th }}, 2014$; Published February 2015

Copyright: @ 2014 IMEKO. This is an open-access article distributed under the terms of the Creative Commons Attribution 3.0 License, which permits unrestricted use, distribution, and reproduction in any medium, provided the original author and source are credited

Funding: (none reported)

Corresponding author: Luca De Vito, e-mail: devito@unisannio.it

\section{INTRODUCTION}

High-speed data acquisition is becoming a relevant topic in advanced applications, such as high-speed radar and communications, signal analysis, high-speed video acquisition, and so on. Moreover, it is a relevant challenge in wideband spectrum sensing for software defined radio and cognitive radio applications [1], [2].

Such demand often is not met by traditional Analog-toDigital Converters (ADCs), due to technological limits in fast sampling rates [3].

The recent studies about compressive sampling (CS) drew a possible solution for signals, that can be represented by a finite number of non-zero elements in a specific domain.

They demonstrated that, for such class of signals, it is possible to reconstruct the original waveform, from a set of samples of a lower dimension than that required by the Shannon theorem.
The idea, underlying the AIC, is to spread the frequency content of the input signal. In this way, the high frequency components, folded back to low frequencies, can be acquired by an ADC with a lower sampling frequency than that required by the Shannon's theorem for the original signal.

Basing on this concept, different architectures have been proposed, implementing the frequency spreading by exploiting: (i) non-uniform [4] or random sampling [5], (ii) random filters [6], and (iii) random demodulation [3].

The aim of the paper is to define performance parameters and test methods for AICs, starting from the state of art of research and the scientific knowledge about ADC testing, well summarized in [7] and [8]. To this aim, the first step is the application to AICs of standard parameters and test methods, actually defined for ADCs in order to study how they are influenced by (i) the AIC architecture type, (ii) the AIC design parameters, and (iii) the circuit non-idealities. 
In the scientific literature few papers can be found, facing the AIC testing and most of them take into account only a reduced set of Figures of Merits (FoMs) and influencing parameters [9].

In [10], the authors presented a preliminary investigation carried out in simulation and on a first AIC prototype, based on a digital oscilloscope, about the application of standard ADC FoMs on the AIC. This paper is an extended version of such work, in which new results are presented and a new AIC prototype is used, based on commercial integrated circuits.

As in [10], the AIC architecture considered in this paper is based on the random demodulation, as it does not require a high sampling frequency ADC. However, test methods and considerations can be easily extended to the other types of AIC architectures.

In particular, in the paper, a characterization of a random demodulation AIC has been carried out by applying a reconstruction algorithm to the AIC output and evaluating the dynamic parameters in the frequency domain, defined for ADC testing. To this aim, in a former phase, a behavioural model of the random demodulation AIC has been simulated, by considering the non-idealities introduced by its main building blocks. In a latter phase, an AIC prototype has been designed, by following the theoretical descriptions found in literature, and an experimental analysis has been conducted on it.

The paper is organized as follows: In Section 2, an introductory description about compressive sampling theory is given; in Section 3, the random demodulation architecture, which was used both in the simulation and the experimental analyses of this work, is described; in Section 4, the approach followed for AIC testing is explained; then, in Section 5, a simulation phase is reported, in which the influence of several factors, such as circuit non-idealities, AIC design parameters and reconstruction algorithm parameters, have been investigated. In Section 6, the FoMs defined for traditional ADCs are revised. Finally, in Section 7 , the experimental analysis is presented and results are discussed.

\section{THEORETICAL BACKGROUND}

The idea underlying CS approach is that many natural signals have concise representations when expressed in a convenient basis [11]. As an example, audio signals have sparse representations in the Short-Time Fourier Transform domain, or in the Modified Discrete Cosine Transform domain [12]. Another example is given by radar echo signals, that, depending on the radar signal type, can have sparse representations in the time, frequency, wavelet, or time-frequency domains [13].

Sparse representations of natural signals, audio, images and videos are currently exploited by transform coding schemes, such as those used by the JPEG, JPEG2000, MPEG, and MP3 standards.

However, in signal compression, signals are acquired using Nyquist rate converters, then they are transformed in a proper domain, where less significant coefficients are discarded.

CS, instead, aims to acquire directly the compressed version of the signal, without wasting acquisition or memory resources, by taking a vector $\mathbf{y}$ of observations of the signal to be acquired, where the size of $\mathbf{y}$ is lower than that required by the Shannon theorem to digitize the signal.

In the past, some other techniques have been proposed to overcome the Shannon theorem constraints in some specific conditions. The equivalent time sampling of time domain signals is an example of such techniques. However, equivalent time sampling requires the observed portion of the signal to be repetitive. CS, instead is applicable even to a non-repetitive signal, providing that a domain can be found, where the representation of such signal is sparse.

For a compressible signal $x(t)$, if $\mathbf{x}$ is a the vector of $N$ samples of it acquired according to the Shannon theorem, its compressed counterpart is represented by a vector $\mathbf{y}$ of size $M<N$, such that:

$\mathbf{y}=\boldsymbol{\Phi} \mathbf{x}$

where, $\Phi$ is a matrix modelling the compression process.

It can demonstrated that an estimate of $\mathbf{x}$ can be reconstructed from $\mathbf{y}$ according to (1) if a matrix transformation $\Psi$ exists, such that:

$\mathbf{x}=\Psi \mathbf{c}$,

where $\mathbf{c}$ has only $K<M$ non-zero elements.

The above defined condition is not rare in reality, since many natural signals are sparse or compressible in the sense that they have concise representations when expressed in the proper basis.

By combining (1) and (2), the following expression is obtained:

$\mathbf{y}=\mathbf{A c}$,

where $\mathbf{A}=\Phi \Psi$ is an $M \times N$ matrix and, therefore, (3) is an under-determined linear system in $\mathbf{c}$.

The system can be solved by finding the solution of (3) that minimizes the $\ell_{0}$ norm, that is having the highest number of non-zero elements in $\mathbf{c}$ :

$\hat{\mathbf{c}}=\operatorname{argmin}\left\{\|\mathbf{c}\|_{0}\right\}$ subject to $\mathbf{y}=\mathbf{A c}$.

The minimization of the $\ell_{0}$ norm is both numerically unstable and NP-complete, requiring an exhaustive enumeration of all $\left(\begin{array}{l}N \\ K\end{array}\right)$ possible locations of the non-zero entries in $\mathbf{c}$ [14]. Therefore, the solution is approximated with that obtained by the minimization of the $\ell_{1}$ norm:

$\hat{\mathbf{c}}=\operatorname{argmin}\left\{\|\mathbf{c}\|_{1}\right\} \quad$ subject to $\mathbf{y}=$ Ac.

In presence of additive noise e, equation (3) becomes:

$\mathbf{y}=\mathbf{A c}+\mathbf{e}$

and the minimization problem is modified as:

$\hat{\mathbf{c}}=\operatorname{argmin}\left\{\|\mathbf{c}\|_{1}\right\}$ subject to $\|\mathbf{y}-\mathbf{A c}\|_{2}<\tau$.

or as:

$\hat{\mathbf{c}}=\operatorname{argmin}\left\{\|\mathbf{c}\|_{1}\right\} \quad$ subject to $\left\|\mathbf{A}^{\mathbf{T}}(\mathbf{y}-\mathbf{A c})\right\|_{\infty}<\varepsilon$. 


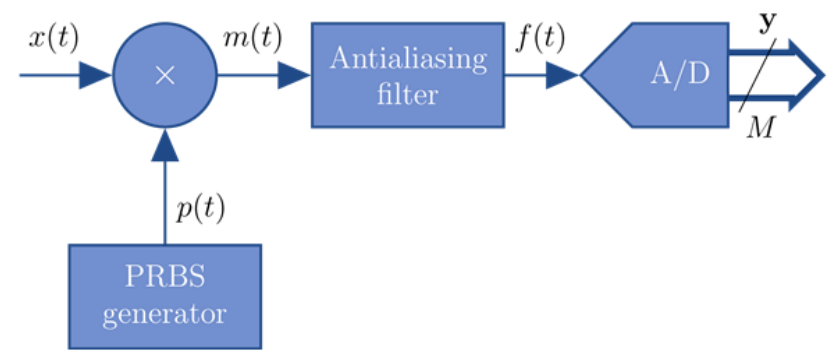

Figure 1: Block scheme of the random demodulation AIC.

where $\tau$ and $\varepsilon$ are small positive constants.

The $\ell_{1}$ norm minimization is a convex optimization problem that conveniently can be reduced to a linear program known as basis pursuit, whose computational complexity is about $O\left(N^{3}\right)$ [14].

The minimum number of observations $M$ required to successfully reconstruct $\mathbf{x}$ is given by the following expression [11]:

$M \geq C \mu^{2}(\Phi, \Psi) K \cdot \log (N)$,

where, $C$ is a positive constant and $\mu(\Phi, \Psi)$ is a quantity, called coherence between $\Phi$ and $\Psi$, having the following expression:

$\mu(\Phi, \Psi)=\sqrt{N} \max _{1 \leq i, j \leq N}\left|\left\langle\varphi_{i}, \psi_{j}\right\rangle\right|$,

with $\varphi_{i}$ and $\psi_{j}$ the $i$-th row of $\Phi$ and the $j$-th column of $\Psi$, respectively, and $\langle\cdot$,$\rangle the dot product.$

The coherence measures the largest correlation between any two elements of $\Phi$ and $\Psi$; If $\Phi$ and $\Psi$ contain correlated elements, the coherence is large; otherwise, it is small [11].

By looking at (9), it can be easily seen that the lower is the coherence between $\Phi$ and $\Psi$, the fewer observations are needed to reconstruct the original signal. It can be demonstrated that incoherence can be achieved with high probability simply by selecting $\Phi$ as a random matrix [14], whose elements are drawn from a suitable distribution, such as Gaussian, Bernoulli or Rademacher.

\section{THE RANDOM DEMODULATION AIC ARCHITECTURE}

The block scheme of the random demodulation AIC is shown in Figure 1. Considering an ADC with sampling frequency $f_{s}$, and a signal $x(t)$, whose frequency content exceeds the first Nyquist region $\left[0, \frac{f_{s}}{2}\right]$. An example of a three-tone signal having such characteristics is shown in Figure 2a. According to the Shannon theorem, it would not be possible to sample $x(t)$, since the components located at frequencies greater than $f_{s} / 2$ would produce aliasing.

It can be observed that for such signal the condition (2) is verified as soon as $\mathbf{x}$ has a Fourier representation with a limited number of non-zero coefficients. In that case, in fact, (2) is simply the Fourier synthesis formula.

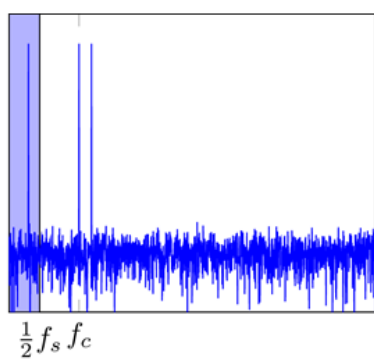

(a) Input signal $x(t)$

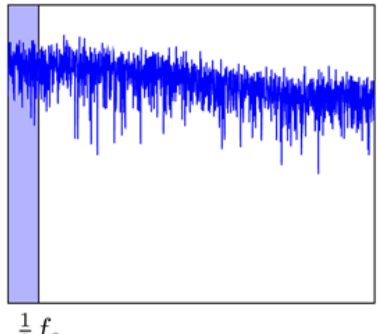

(c) Mixer output $m(t)$

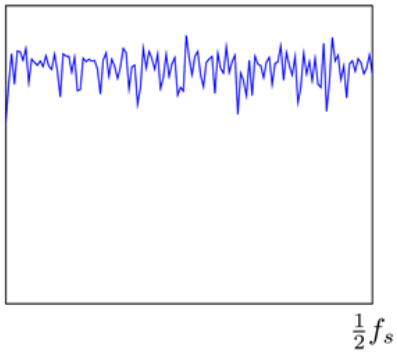

(e) Observed signal

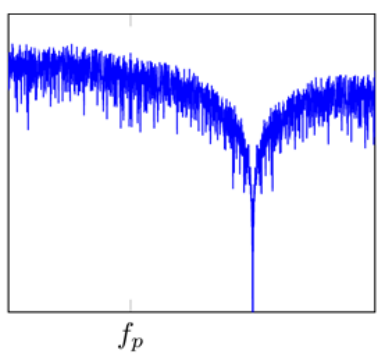

(b) PRBS $p(t)$

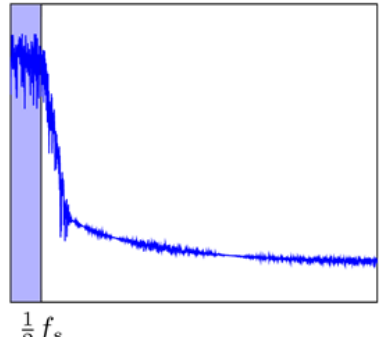

(d) Filter output $f(t)$

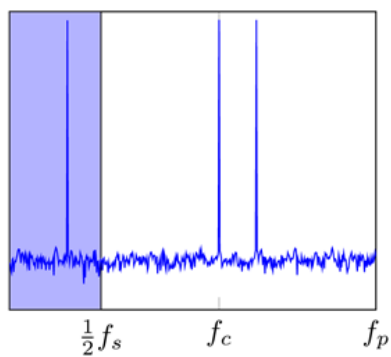

(f) Reconstructed signal
Figure 2. Spectra of the signals processed inside the AIC structure. The AIC is able of reconstructing a signal even if its spectral content exceeds the first Nyquist region.

Therefore, first, a compressed version of $\mathbf{x}$ is obtained, and, then, the signal could be reconstructed by solving one of the problems (5), (7) or (8).

In order to obtain the compressed version of $x(t)$, it is mixed with a Pseudo-Random Binary Sequence (PRBS), having a bit rate equal to $f_{p}=\mathcal{M} f_{s}$, with $\mathcal{M}$ positive integer greater than 1 . The bit rate of the PRBS determines the maximum frequency component of the original signal that the AIC is able to acquire, as it is required to be at least twice the maximum frequency component of the signal.

The spectrum of the PRBS, shown in Figure $2 b$, has a frequency content spread over the whole region $\left[0, f_{p}\right]$, therefore, the output of the mixer contains the information of the original signal, spread over the whole first Nyquist band, even if it was originally located outside such region (Figure 2c). This allows that after low-pass filtering (Figure 2d) and A/D conversion (Figure 2e), the information contained in the original signal has not been lost. However, it needs to be recovered from the filter output.

A reconstruction algorithm is, then, in charge of extracting this information, by solving one of the problems in (5), (7) or (8), as previously quoted, giving thus a digital representation of the original signal (Figure 2f). 
The capability of the reconstruction algorithm of actually recovering the original signal with high probability depends on the undersampling factor $\mathcal{M}$, and on the sparsity rate $K / M$. A performance analysis for a random demodulator using an integrate and dump filter has been carried out in [15], and the results showed that a high probability of reconstruction could be achieved if:

$$
M \geq 1.7 K \log \left(\frac{N}{K}+1\right)
$$

Therefore, the AIC is not able to preserve the information contained in the signal if undersampling is below a certain value, depending on the number of non-zero element in the sparse representation of the signal to be acquired.

The architecture can be extended by using several parallel channels, each driven by a different PRBS. In this case, the AIC is capable of acquiring signals even in the case undersampling causes the overlapping of several signal components, since in each channel, the overlapping appears in different form [16]. For sake of simplicity, in this paper the analysis has been conducted on a single channel architecture. However, results can be easily extended to a multi-channel architecture.

A mathematical model of the AIC can be obtained as it follows. Indicating with $\mathbf{x}$ the vector of the signal samples, if it was acquired using a sampling frequency equal to the bit rate of the PRBS $f_{p}$, and with $\mathbf{p}=\left[\begin{array}{llll}p_{0}, & p_{1}, & \ldots, & p_{N-1}\end{array}\right]$ the samples of the PRBS, the output of an ideal digital mixer $\mathbf{m}$ can be obtained by multiplying $\mathbf{x}$ by a diagonal matrix, having the PRBS samples on the diagonal:

$$
\mathbf{m}=\mathbf{D x}
$$

where:

$$
\mathbf{D}=\left[\begin{array}{llll}
p_{0} & 0 & \cdots & 0 \\
0 & p_{1} & \cdots & 0 \\
\vdots & \vdots & \ddots & \vdots \\
0 & 0 & \cdots & p_{N-1}
\end{array}\right]
$$

Instead, the filter can be modelled by the following Toeplitz matrix:

$$
\mathbf{H}=\left[\begin{array}{lllllll}
h_{1} & h_{2} & \cdots & h_{L-1} & 0 & \cdots & 0 \\
0 & h_{1} & \cdots & h_{L-2} & h_{L-1} & \cdots & 0 \\
\vdots & \vdots & \ddots & \vdots & \vdots & \cdots & \vdots \\
0 & 0 & \cdots & \cdots & \cdots & h_{L-2} & h_{L-1}
\end{array}\right]
$$

where, $\mathbf{h}=\left[\begin{array}{llll}h_{0} & h_{1} & \cdots & h_{L-1}\end{array}\right]^{T}$ is the truncated impulse response of the antialiasing filter of length $L$. $L$ should be chosen large enough that the truncated response guarantees a good approximation of the actual one.

The random demodulation AIC can be obtained by choosing a $\Phi$ matrix that decimates the matrix product H D, by taking only 1 every $\mathcal{M}$ rows.

\section{APPROACH TO AIC TESTING}

AIC testing is a harder task than traditional ADC testing, due to the following reasons: (i) the AIC output signal has a strong stochastic behaviour, due to the PRBS (compare Figs.2a and 2e); (ii) the AIC output signal is noiselike for every input, thus, it is hard to analyse either in the time or in the frequency domain; and (iii) the parameters of the reconstruction algorithm can affect the AIC results.

The current research has been focused on frequencydomain testing, as it is mostly used in telecommunications, which is the main application field of AICs. The approach followed for AIC testing consists of using a sinewave signal as input to the AIC, applying a reconstruction algorithm, and characterizing the spectrum of the reconstructed signal, by means of the traditional ADC FoMs. Test signal frequencies have been chosen such that the coherent sampling condition is respected for the reconstructed signal, that is they should verify the following condition:

$\omega_{0}=\frac{J}{N} 2 \pi$,

where, $J$ is an integer number, relatively prime to $N$.

The ADC testing methods in the frequency domain can be clearly applied to AIC as the involved signals are sparse in such domain. As sparsity in the frequency domain has been considered, the $\Psi$ matrix in (2) must represent a transformation between time domain and frequency domain. However, since only real-valued test signals have been considered, the Discrete Hartley Transform (DHT) basis has been used, which is equivalent to the Discrete Fourier Transform, but is purely real.

The generic element $\psi_{i j}$ of the DHT has the following form:

$\psi_{i, j}=\frac{1}{\sqrt{N}}\left[\cos \left(\frac{2 \pi}{N} i j\right)+\sin \left(\frac{2 \pi}{N} i j\right)\right]$.

The Dantzig selector [17] has been used as reconstruction algorithm, solving the optimization problem in (8), by means of the implementation given by the $\ell_{1}$-magic MATLAB toolbox [18].

Differently from problem (7), which looks for the solution in the least square sense, the Dantzig selector computes the residual $\mathbf{r}=\mathbf{y}-\mathbf{A} \mathbf{c}$, and, then, evaluates its inner products with the columns of $\mathbf{A}$. These inner products form various weighted combinations of the entries in $\mathbf{r}$ and all those are expected to be small [19]. The constraint, thus, proceeds by requiring that all these are below the threshold $\varepsilon$.

Therefore, the parameter $\varepsilon$ represents the maximum allowable value for the correlation of the residual $\mathbf{r}$ with any column of $\mathbf{A}$.

In a preliminary phase, the AIC has been modelled and some simulations have been carried out in order to understand how the FoMs, evaluated on the reconstructed signal, can be affected by: (i) circuit non-idealities, (ii) test signal parameters, and (iii) reconstruction algorithm parameters. 


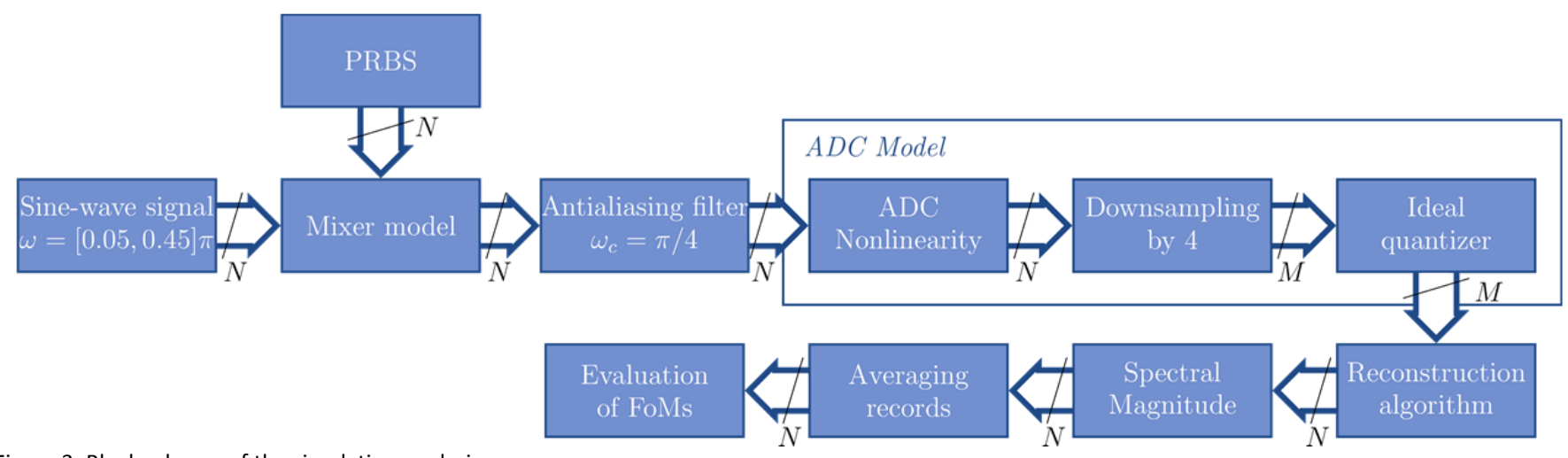

Figure 3: Block scheme of the simulation analysis.

According to the results of the simulation analysis, the following FoMs, have been extended to AICs: Spurious Free Dynamic Range (SFDR), Signal to Noise and Distortion Ratio (SINAD), and Total Harmonic Distortion (THD).

For such FoMs, the definitions in [20] have been used. However, the same approach can be used even considering definitions coming from different standards, such as those considered in [21].

Finally, an experimental phase has been conducted by evaluating the revised FoMs on a specifically designed AIC prototype.

\section{SIMULATION ANALYSIS}

During simulation analysis, an explicit behavioural model [22] has been considered for each AIC block.

In Figure 3, the steps carried out for the simulation analysis have been drawn. Sinewave signals have been generated, with frequency in the range [0.05-0.45] $\pi$, with a record length of $N=512$ samples. Signal records are then given to a mixer model, together with PRBS records of the

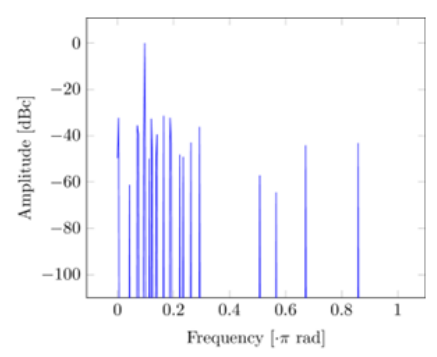

a)

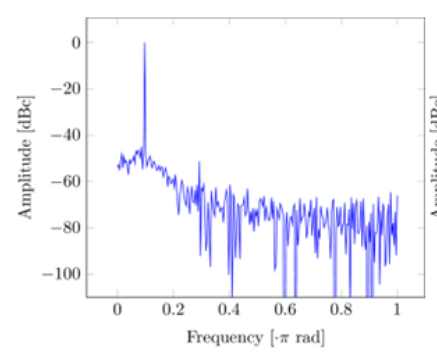

c)

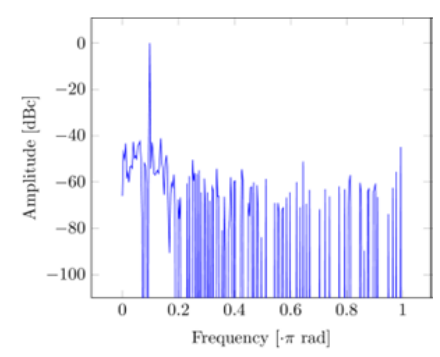

b)

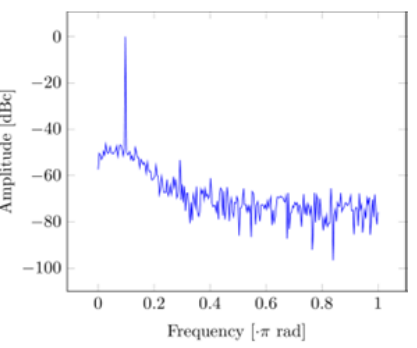

d) same size.

As any actual mixer will distort the signals, the ideal mixer output $y_{i d}=x_{1}(t) x_{2}(t)$ (see Eq. 12) has been replaced by a second order polynomial model, as follows:

$y(t)=a x_{1}(t)+b x_{2}(t)+x_{1}(t) x_{2}(t)+c x_{1}^{2}(t)+d x_{2}^{2}(t)$

where, $x_{1}(t)$ and $x_{2}(t)$ are the inputs to the mixer, respectively, and $a, b, c$ and $d$ are attenuation coefficients.

The output of the mixer is given to a lowpass FIR filter, with cut-off frequency equal to $\pi / 4$, modelling the antialiasing filter.

The ADC, embedded in the AIC architecture, has been modelled as a memoryless nonlinearity followed by a downsampling by 4 and an ideal quantizer, where the nonlinearity has been obtained, as in [9], using the following third order polynomial function:

$y(t)=\frac{x(t)+c_{3} x^{3}(t)}{1+c_{3}}$

Due to downsampling, the record length is reduced to $\mathrm{M}=128$ samples. The output of the ADC model is given to the reconstruction algorithm, able of regenerating a record of $\mathrm{N}=512$ samples containing the reconstructed signal.

Finally, the FoMs are evaluated on the averaged spectral magnitude of the reconstructed signal. Averaging of spectral magnitude is specified by [20] for the evaluation of the FoMs in the frequency domain. However, as it is shown in Section 5.1, a greater average number is needed than in the case of ADCs.

The above described steps have been executed on several signals, when the parameters of the models have been varied as shown in the following Subsections.

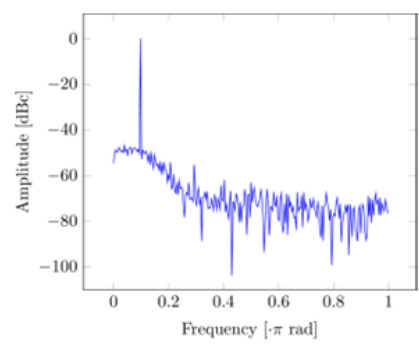

a)

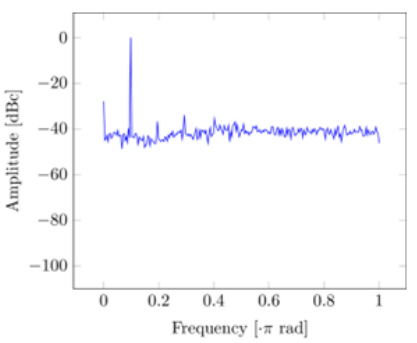

b)
Figure 4. Spectral magnitudes obtained by the FFT magnitude on a single record (a), or by averaging the FFT magnitudes evaluated on 10 (b), 100 (c), and 200 (d) records.
Figure 5. Averaged spectral magnitudes of the reconstructed signal without mixer nonlinearity (a), and with mixer nonlinearity (b). 


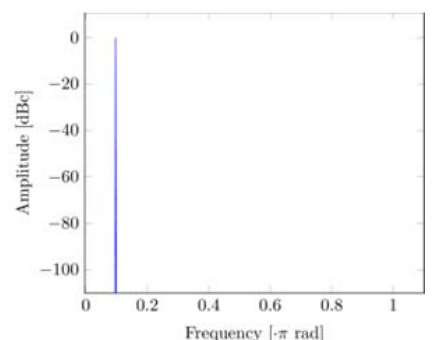

a)

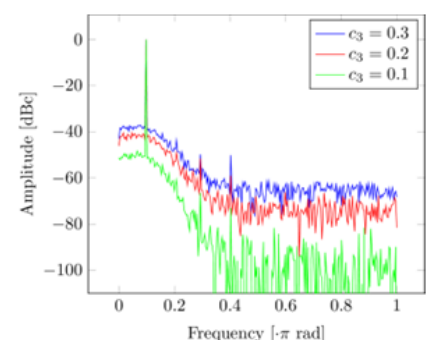

b)
Figure 6. Averaged spectral magnitudes of the reconstructed signal without $\mathrm{ADC}$ nonlinearity and with a nonlinearity coefficient $c_{3}$ equal to $0.1,0.2$, and 0.3 , respectively.

\subsection{Influence of average number}

In Figure 4, the spectral magnitude is shown, when it is obtained by the FFT (Fast Fourier Transform) magnitude of a single record (Figure 4a), or when it is obtained by averaging FFT magnitudes evaluated on different records. Results are shown for a number of averages equal to 10 (Figure 4b), 100 (Figure 4c), and 200 (Figure 4d). As it can be noted, due to the selection of frequency components operated in the reconstruction algorithm, some of them are not identified in a single record. Instead, the averaging allows obtaining an estimation of the spectral magnitude for each frequency component.

Such behaviour is more evident for higher values of $\varepsilon$, since in this case a higher number of components are discarded by the reconstruction algorithm.

\subsection{Influence of mixer nonlinearity}

The main effect of the mixer nonlinearity is of increasing the level of the noise floor, as shown in Figure 5, where the averaged spectral magnitudes of the reconstructed signal when no mixer nonlinearity has been modelled $(a=b=c=d=0$ ) (Figure5a), and when a nonlinearity is added, with the following values of the attenuation coefficients: $a=b=0.1, c=d=0.01$ (Figure5b).

Those values have been chosen by looking at the order of magnitude of the typical feedthrough and second order distortion component attenuation for actual mixers.

In addition, a particular increase of the level of the components, whose frequencies lie at multiples of the fundamental, has been observed.

\subsection{Influence of $A D C$ nonlinearity}

The effect of ADC nonlinearity can be observed in Figure 6, where the averaged spectral magnitudes of the reconstructed signals are reported in the case the $c_{3}$ parameter is set to 0 (Figure 6a), simulating a linear ADC, and when the $c_{3}$ parameter is set to 0.2 (Figure 6b). Differently from traditional ADCs, the nonlinearity does not act only on a restricted number of components, located at multiples of the fundamental, in fact it causes even an

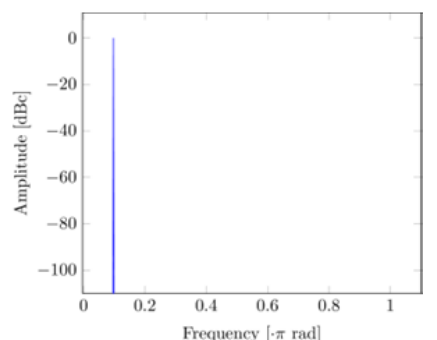

a)

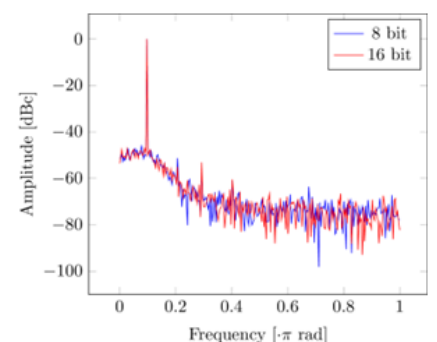

b)
Figure 7. Averaged spectral magnitudes of the reconstructed signal when infinite resolution is used (a), and when a quantization of 8 bit or 16 bit is used (b).

increase of the noise floor.

\subsection{Influence of $A D C$ quantization}

The ADC quantization causes an increase of the noise floor level. As it can be seen in Figure 7a, the signal is perfectly reconstructed (with noise floor level depending only on the machine precision) when maximum machine resolution is used. Instead, a noise floor arises when quantization is used. However, only a small difference in the level has been observed for different resolutions. During simulation analysis, values of the resolution in the set $\{8$, $10,12,14$, and 16$\}$ bits have been used. In Figure $7 \mathrm{~b}$, the averaged spectral magnitudes, related to a resolution of 8 bits and 16 bits are shown.

\subsection{Influence of reconstruction parameter $\varepsilon$}

As the reconstruction parameter $\varepsilon$ represents a limit to the amount of additive noise that can be tolerated on the output signal, it controls the selection of the frequency components in the reconstructed signal. In fact, for each record, a frequency component will be included in the reconstructed signal, only if its energy exceeds a certain level, depending on the value of $\varepsilon$.

On the averaged spectrum, this causes an increase of the level of both the noise floor and of the harmonics, for decreasing values of $\varepsilon$.

Such behaviour can be observed in Figure 8a, where the averaged spectral magnitudes of the reconstructed signal are shown, where $\varepsilon$ has been set to $0.01,0.05$, and 0.1 .

\subsection{Influence of the filter length}

The length of the impulse response of the anti-aliasing filter determines the capability of the filter of attenuating the aliasing replicas. The shorter the filter impulse response is, the lower such capability is. Figure $8 \mathrm{~b}$ highlights this behaviour, by showing the averaged spectral magnitudes of the reconstructed signals, when filter lengths of 17 and 49 taps are used. As it can be noted, in the case of a filter length of 17 (blue line), the filter is not able to adequately attenuate the aliasing replicas, located at the frequencies

$T H D=20 \log _{10} \sqrt{\frac{\sum_{h=2}^{N_{h}} X_{a v m}^{2}\left[n_{h}\right]+\sum_{h=-N} X_{h v m}^{2}\left[n_{h}\right]+X_{a v m}^{2}\left[M-n_{i} \mid\right]+X_{a v m}^{2}\left[\bmod \left(M+n_{i, 2}\right)\right]+X_{a v m}^{2}\left[N-\left|M-n_{i}\right|\right]+X_{a v m}^{2}\left[N-\bmod \left(M+n_{i, 2}\right)\right]}{X_{a v m}^{2}\left[n_{i}\right]+X_{a v m}^{2}\left[M-n_{i}\right]}}$ 


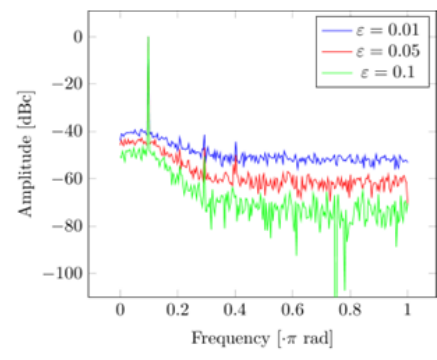

a)

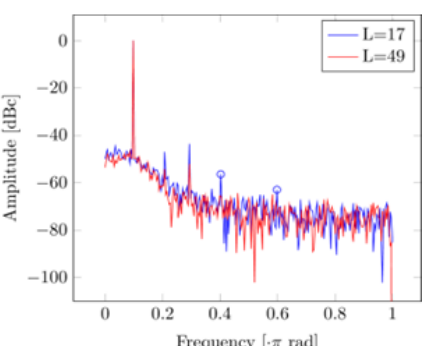

b)
Figure 8. Averaged spectral magnitudes of the reconstructed signal for values of $\varepsilon$ equal to $0.01,0.05$, and 0.1 . (a), respectively, and the averaged spectral magnitudes of the reconstructed signal, for filter lengths equal to 17 and 49 taps (b).

$\left\{\frac{2 \pi}{\mathcal{M}}-\omega_{0}, \frac{2 \pi}{\mathcal{M}}+\omega_{0}\right\}$.

In the case the filter length is of 49 taps (red line), instead, the aliasing components are well attenuated under the noise floor.

\section{DEFINITION OF THE FIGURES OF MERIT}

As previously mentioned, the FoMs used in this work have been defined starting from those of the IEEE Std. 1241-2010 [20]. However, the following modifications have been introduced: (i) the number of averages of the spectral magnitudes has been set to 200 , in order to take into account the stronger stochastic behaviour of the reconstructed signal, and (ii) the frequencies $\left\{\frac{2 \pi}{\mathcal{M}}-\omega_{0}, \frac{2 \pi}{\mathcal{M}}+\omega_{0}\right\}$ have been included in the list of the harmonics, contributing to the THD.

The FoMs have been evaluated by averaging the spectral magnitude $\mid X[m]$ as it follows [20]:

$X_{\text {avm }}[m]=\frac{1}{R} \sum_{k=1}^{R} \mid X_{k}[m], \quad m=0,1,2, \ldots, N-1$,

over a number of $R$ data records.
Compared to definitions in [20], no changes have been made to the SFDR and SINAD:

$$
\begin{aligned}
& S F D R=20 \log _{10}\left(\frac{\mid X_{\text {avm }}\left[n_{i}\right]}{\max _{m \in S_{0}} \mid X_{a v m}[m]}\right) \\
& S I N A D=20 \log _{10} \sqrt{\frac{N-3}{N} \cdot \frac{X_{a v m}^{2}\left[n_{i}\right]+X_{a v m}^{2}\left[N-n_{i}\right]}{\sum_{m \in S_{0}} X_{a v m}^{2}[m]}}
\end{aligned}
$$

where, $n_{i}$ is the frequency index corresponding to the fundamental component, $S_{0}$ is the set of frequency indexes from 1 to $N-1$, excluding the two values corresponding to the fundamental and its image.

Instead, THD has been modified as shown in (22), where, $N_{h}$ is the number of the highest harmonics considered, $\bmod (\cdot, \cdot)$ is the modulo operator, and $n_{h}$ is the frequency index corresponding to a generic harmonic or its aliasing component:

$$
n_{h}=\bmod \left(h n_{i}, N\right) \quad h= \pm\left[2,3, \ldots, N_{h}\right]
$$

In the considered case $N_{h}$ has been chosen equal to 10 .

\section{EXPERIMENTAL INVESTIGATION}

For the purpose of the experimental investigation, an AIC prototype has been designed, using commercially available circuits and bench instrumentation. In the following Subsections, the AIC prototype is described, then, some details, about the calibration phase needed to estimate the $\Phi$ matrix, are given; finally, the experimental results are presented.

\subsection{The AIC prototype}

A block scheme of the AIC prototype is shown in Figure 9. The mixing process is carried out by means of the Analog Devices AD8342 evaluation board, while the antialiasing filter has been realized by the first channel of the Analog Devices ADRF6510 evaluation board. The ADRF6510 is a dual-channel programmable lowpass filter, with cut-off frequency tunable in the range [1-30] $\mathrm{MHz}$.

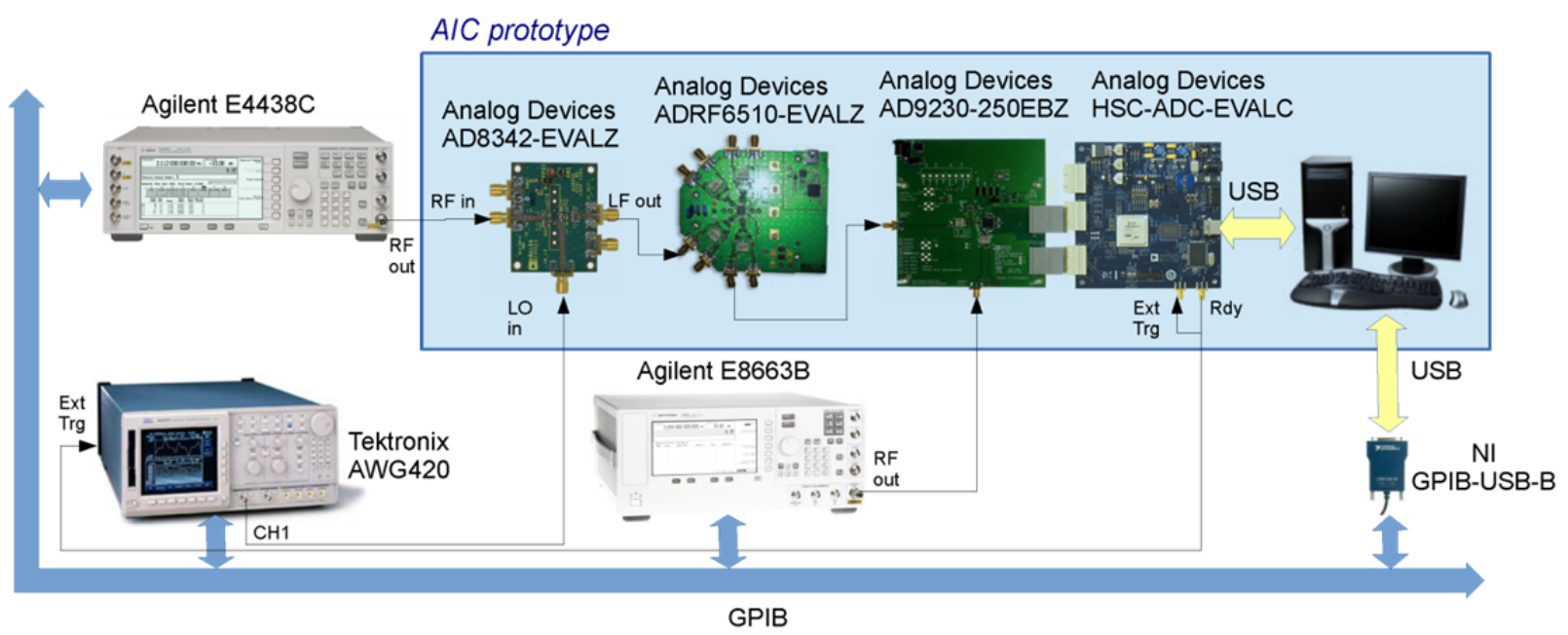

Figure 9. Block scheme of the AIC prototype and the test setup, used during experimental analysis. 

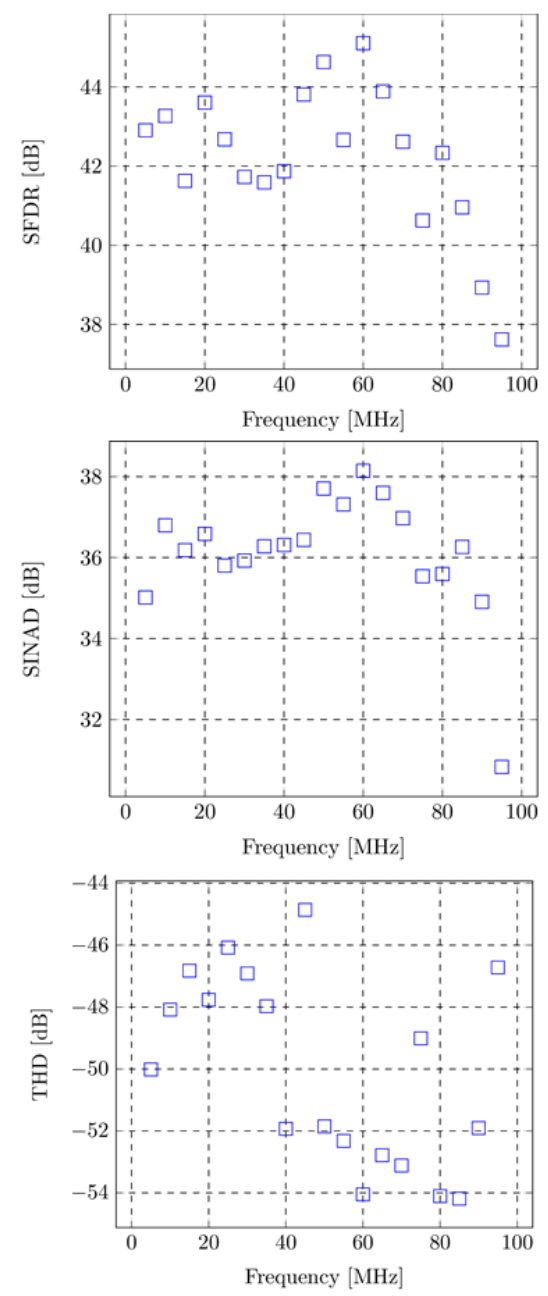

Figure 10. Experimental results for test signal frequency ranging from 5 to $95 \mathrm{MHz}: \operatorname{SFDR}(\mathrm{a}), \operatorname{SINAD}(\mathrm{b})$, and THD (c), for $\varepsilon=0.5$.

After mixing and filtering, signal is acquired by means of an Analog Devices AD9230 evaluation board, operating at a sampling frequency of $50 \mathrm{MS} / \mathrm{s}$. The ADC board is connected with a computer through an Analog Devices HSC-ADC-EVALC Capture board.

For the test purpose, sinewave signals have been generated by an Agilent E4438C Vector Signal Generator, while the PRBS is generated in the computer and downloaded, through the GPIB, to the Tektronix AWG420 Arbitrary Waveform Generator. An Agilent E8663B is used to generate the sampling clock for the ADC board.

In order to synchronize the PRBS generation with the acquisition, the HSC-ADC-EVALC Capture board has been configured with a specific firmware allowing external triggering. In this case, the acquisition is started on the computer, causing a Ready signal to be set on the HSCADC-EVALC Capture board. Such Ready signal is given both to the Tektronix AWG420, to generate the PRBS and again to the HSC-ADC-EVAL Capture board to start the acquisition. The Agilent E4438C, the Tektronix AWG420 and the Agilent E8663B have been then synchronized by means of a $10 \mathrm{MHz}$ reference, in order to guarantee the coherent sampling condition, and controlled by the computer by means of a GPIB interface.

\section{2. $\Phi$ matrix estimation}

In order to run the reconstruction algorithm, it is necessary to estimate the $\Phi$ matrix in (1). Since it is obtained from the PRBS and from the impulse response of the anti-aliasing filter through (13) and (14), only the estimation of the impulse response of the anti-aliasing filter is necessary.

This is done in a preliminary calibration phase, by connecting the Tektronix AWG420, generating a PRBS, directly to the input of the filter. Then, the output of the filter is acquired. As shown in [23], if the input to a system $x(t)$ is a stochastic process, having impulsive autocorrelation function, the impulse response of the system can be measured as:

$h(n)=\frac{r_{x y}(n)}{r_{x x}(0)}$,

where, $r_{x y}(n)$ is the cross-correlation function between the input and the output and $r_{x x}(0)$ is the autocorrelation of the input, evaluated at a lag equal to 0 , corresponding to the input signal energy. Since in the considered case the generated PRBS signal has impulsive autocorrelation, it is possible to estimate the impulse response of the filter using (24).

\subsection{Experimental results}

Using the AIC prototype and the test setup shown in Figure 9, a wide experimental investigation has been carried out, by evaluating the SFDR, SINAD, and THD, on the reconstructed signal. As in simulation tests, sinewave input signals have been used. In order to guarantee the coherent sampling condition, the test signal frequency has been selected, using the following expression:

$f_{0}=\frac{J}{N} f_{p}$

where, $f_{p}$ is the PRBS bit rate, corresponding to the equivalent sampling frequency, and $J$ is an integer coprime to $N$.

Sinewave signals have been generated with frequencies in the range [5 - 95] MHz and PRBS bit rate has been fixed to $200 \mathrm{MHz}$. Different values of the cut-off frequency of the antialiasing filter have been used in the range $[10-30] \mathrm{MHz}$, while the sampling frequency of the ADC embedded in the AIC architecture has been set to $50 \mathrm{MS} / \mathrm{s}$.

Since the band of the reconstructed signal is in the range [0-100 MHz], and the Nyquist frequency of the ADC embedded in the AIC is $25 \mathrm{MHz}$, the AIC allows extending 4 times the signal bandwidth, compared with the case of the $\mathrm{ADC}$ embedded in the AIC architecture, running at the same sampling frequency.

Some results of the experimental investigation are shown in Figure 10, for a test signal frequency in the range [5-95] MHz, with a step of $5 \mathrm{MHz}$.

The variations of the values of all three FoMs are in the range of some $\mathrm{dBs}$, even beyond the first Nyquist band [0-25] $\mathrm{MHz}$, however, performance show a little decrease, in SFDR and SINAD, for frequencies approaching 100 $\mathrm{MHz}$. 

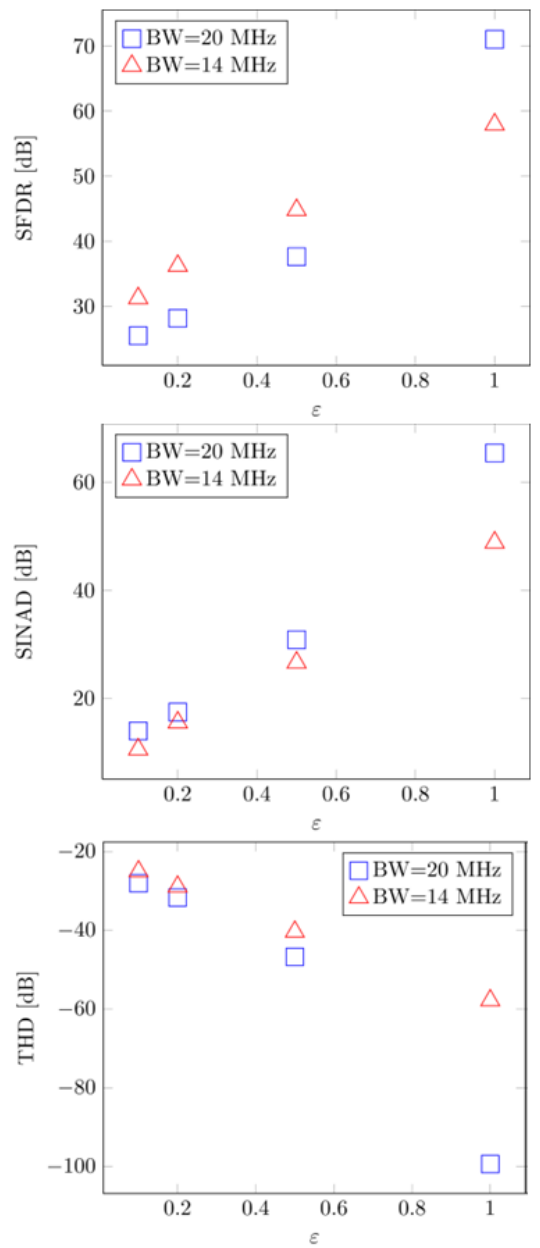

Figure 11. Evaluated FoMs versus $\varepsilon$ and filter bandwidth, for a test signal frequency of $10 \mathrm{MHz}$.

It is worth studying the variation of the FoMs versus the reconstruction parameter $\varepsilon$, as well as versus the bandwidth of the anti-aliasing filter.

As shown by the simulation results, the levels of both the noise floor and the harmonic components decrease with $\varepsilon$, therefore, an increase in the values of SINAD and SFDR is expected, while THD is expected to decrease. This can be easily observed in Figure 11, where the trend of the

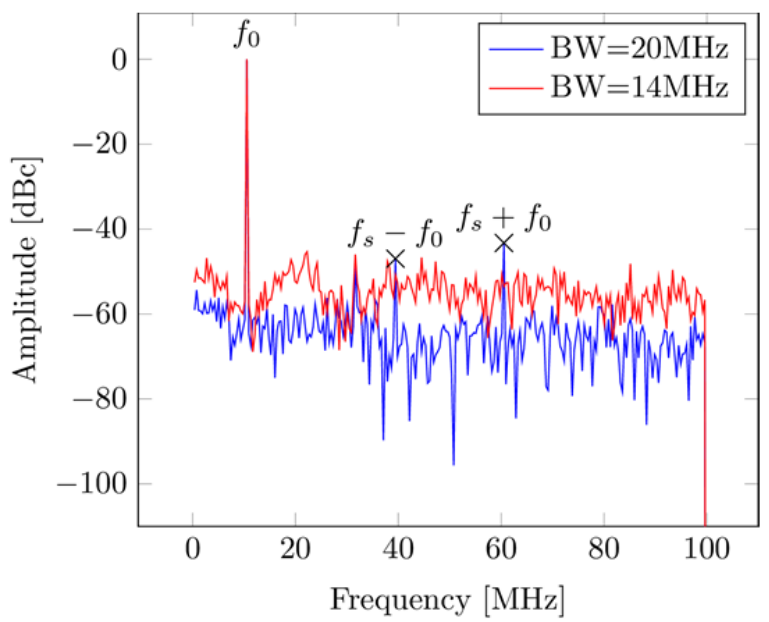

Figure 12. Averaged spectral magnitude of the reconstructed signal for filter bandwidth equal to 14 and $20 \mathrm{MHz}$. considered FoMs versus $\varepsilon$ is shown.

In Figure 11, the variation of the FoMs versus the bandwidth of the anti-aliasing filter is shown, too. It can be observed that the values of SINAD and THD are generally better for a filter with a wider band. This is probably due to the fact that a greater amount of information passes through the filter and can be used for the reconstruction. In the case of the SFDR, instead, smaller values have been observed with the narrowband filter, and $\varepsilon<1$. As it can be seen in Figure 12, this due to the aliasing components at $f_{s}-f_{0}$ and $f_{s}+f_{0}$, that are not attenuated under the noise floor level, by the $20 \mathrm{MHz}$ bandwidth filter. In the case of a $20 \mathrm{MHz}$ bandwidth, in fact, the noise floor level is lower, but the aliasing component are higher than the case of a $14 \mathrm{MHz}$ bandwidth.

\section{CONCLUSIONS AND FURTHER WORK}

In this paper, a frequency-domain characterization of AICs has been presented, by concentrating the attention on the random demodulation architecture. In a former phase, the AIC has been simulated by means of explicit behavioural models of its main components, in order to evaluate the influence of the circuit non-idealities, the design parameters and the reconstruction algorithm to the FoMs, currently defined for ADCs.

The simulation results show that both mixer and ADC nonlinearities contribute to increase the noise floor level, as well as the level of the harmonic components. This is quite different from what happens in traditional ADCs, where nonlinearities do not affect the noise floor level. In addition, the antialiasing filter can affect the SFDR, as it can not completely attenuate the aliasing components. As result of the simulation analysis, a modification to the THD definition is proposed by including the energy of the aliasing components.

In a latter phase, an experimental analysis has been conducted on a specifically designed AIC prototype, using sinewaves, by varying the test input frequency, the reconstruction parameter $\varepsilon$, and the bandwidth of the antialiasing filter. Such analysis showed that the proposed FoMs are in general capable of describing the performance of the AIC. However, the FoMs should be observed for different values of $\varepsilon$, since high values of $\varepsilon$ can hide the effects of noise and distortion.

Further work will be directed to analyse the Intermodulation Distortion as frequency domain FoMs. Then, it can be interesting to observe the FoMs even evaluated in the time domain. In addition, the methodology presented in this paper can be extended to other AIC architectures, such as those based on the random sampling. Finally, an alternative approach to AIC testing can be used, without relying on the reconstruction algorithm, but directly comparing the AIC output, with the expected output of the AIC model. 


\section{REFERENCES}

[1] L.De Vito, "Methods and technologies for wideband spectrum sensing”, Measurement, vol.46, No.9, Nov.2013, pp. 3153-3165.

[2] D.Bao, L.De Vito, S.Rapuano, “A histogram-based segmentation method for wideband spectrum sensing in cognitive radios", IEEE Trans. on Instrum. and Meas., vol. 62, No.7, July 2013, pp. 1900-1908.

[3] S.Kirolos, J.Laska, M.Wakin, M.Duarte, D.Baron, T.Ragheb, Y.Massoud, R.Baraniuk, "Analog-to-information conversion via random demodulation”, Proc. of 2006 IEEE Dallas/CAS Workshop on Design, Applications, Integration and Software, Richardson, US, Oct. 2006, pp. 71-74.

[4] M.Wakin, S.Becker, E.Nakamura, M.Grant, E.Sovero, D.Ching, J.Yoo, J.Romberg, A.Emami-Neyestanak, E.Candes, "A nonuniform sampler for wideband spectrallysparse environments", IEEE Journal on Emerging and Selected Topics in Circuits and Systems, vol.2, No.3, Sept. 2012, pp. 516-529.

[5] J.Laska, S.Kirolos, "Random sampling for analog-toinformation conversion of wideband signals", Proc. of IEEE Dallas/CAS Workshop on Design, Applications, Integration and Software, Richardson, US, Oct. 2006, pp. 119-122.

[6] J.A.Tropp, M.B.Wakin, M.F.Duarte, D.Baron, R.G.Baraniuk, "Random filters for compressive sampling and reconstruction", Proc. of IEEE Int. Conf. on Acoustics Speed and Signal Processing, Toulouse, France, May 2006, vol.3, pp. III-872-III-875.

[7] S.Rapuano, P.Daponte, E.Balestrieri, L.De Vito, S.J.Tilden, S.Max, J.Blair, "ADC parameters and characteristics - Part 6 in a series of tutorials in instrumentation and measurement", IEEE Instrum. Meas. Magazine, vol.8, No.5, 2005, pp. 44-54.

[8] T.E.Linnenbrink, J.Blair, S.Rapuano, P.Daponte, E.Balestrieri, L.De Vito, S.Max, S.J.Tilden, "ADC testing Part 7 in a series of tutorials in instrumentation and measurements”, IEEE Instrum. Meas. Magazine, vol.9, No.2, 2006, pp. 39-49.

[9] Z.Yu, J.Zhou, M.Ramirez, S.Hoyos, B.M.Sadler, "The impact of ADC nonlinearity in a mixed-signal compressive sensing system for frequency-domain sparse signals", Physical Communication, vol.5, No.2, June 2012, pp. 196-207.
[10] D.Bao, P.Daponte, L.De Vito, S.Rapuano, "Defining frequency domain performance of Analog-to-Information converters", Proc. of 17th IMEKO Int. Workshop on ADC Modelling and Testing, Barcelona, Spain, July 2013, pp. 748-753.

[11] E.J.Candes, M.B.Wakin, "An introduction to compressive sampling”, IEEE Signal Processing Magazine, vol.25, March 2008, pp. 21-30.

[12] B.M.D.Plumbley, T.Blumensath, L.Daudet, M.E.Davies, "Sparse representations in audio and music: from coding to source separation," Proc. of the IEEE, V.ol.98, No.6, June 2010, pp.995-1005.

[13] Z.Lei, Q.Chunting, "Application of compressed sensing theory to radar signal processing", Proc. of 3rd Int. Conf. Comput. Sci. Inf. Technol., Jul. 2010, pp. 315-318.

[14] R.G.Baraniuk, "Compressive Sensing [Lecture Notes]", IEEE Signal Processing Magazine, vol.24, N.4, July 2007, pp.118121.

[15] J.Tropp, J.Laska, "Beyond Nyquist: Efficient sampling of sparse bandlimited signals," IEEE Trans. Inf. Theory, vol.56, No.1, 2010, pp.520-544.

[16] M.Mishali, Y.Eldar, "Sub-Nyquist acquisition hardware for wideband communication", Proc. of IEEE Workshop on Signal Processing Systems (SIPS), 2010, pp. 156-161.

[17] E.Candes, T.Tao, “The Dantzig selector: Statistical estimation when $\mathrm{p}$ is much larger than n", The Annals of Statistics, vol.35, No.6, Dec.2007, pp. 2313-2351.

[18] $\ell 1$-magic toolbox, http://users.ece.gatech.edu/ justin /11magic/.

[19] M.Elad, "Sparse and redundant representations: from theory to applications in signal and image processing", Springer, New York, 2010.

[20] "IEEE Standard for terminology and test methods for analogto-digital converters", IEEE Std. 1241, 2011.

[21] S.Rapuano, "Preliminary considerations on ADC standard harmonization”, IEEE Trans. on Instrum. and Meas., vol.57, No.2, Feb.2008, pp. 386-394.

[22] P.Arpaia, P.Daponte, S.Rapuano, "A state of the art on ADC modelling”, Computer Standards and Interfaces, vol.26, No.1, Jan.2004, pp. 31-42.

[23] K.J.Keesman, "System identification: an introduction", Springer-Verlag, London, 2011. 